\title{
Computer Assisted REhabilitation (CARE) Lab: A novel approach towards Pediatric Rehabilitation 2.0
}

\author{
Ivana Olivieri*, Paolo Meriggi, Cristina Fedeli, Elena Brazzoli, Anna Castagna, \\ Marina Luisa Rodocanachi Roidi and Lucia Angelini \\ IRCCS Fondazione Don Carlo Gnocchi, Milan, Italy
}

Accepted 17 August 2017

\begin{abstract}
Pediatric Rehabilitation therapists have always worked using a variety of off-the-shelf or custom-made objects and devices, more recently including computer based systems. These Information and Communication Technology (ICT) solutions vary widely in complexity, from easy-to-use interactive videogame consoles originally intended for entertainment purposes to sophisticated systems specifically developed for rehabilitation.

This paper describes the principles underlying an innovative "Pediatric Rehabilitation 2.0" approach, based on the combination of suitable ICT solutions and traditional rehabilitation, which has been progressively refined while building up and using a computer-assisted rehabilitation laboratory. These principles are thus summarized in the acronym EPIQ, to account for the terms Ecological, Personalized, Interactive and Quantitative. The paper also presents the laboratory, which has been designed to meet the children's rehabilitation needs and to empower therapists in their work. The laboratory is equipped with commercial hardware and specially developed software called VITAMIN: a virtual reality platform for motor and cognitive rehabilitation.
\end{abstract}

Keywords: Pediatric rehabilitation, virtual reality

\section{Introduction}

Pediatric rehabilitation (PR) focuses on children along their different phases of growth, and thus it has to take into account their stage of development and emerging needs. PR primary goal is the improvement of impaired abilities (and sometimes recovering those that have been lost), with the ultimate aim of providing individualized opportunities for "full and effective participation and inclusion in society" [1]. This process should not be confined to rehabilitation centers but transition gradually into the children's everyday life, involving their caregivers, teachers, relatives and friends [2].

\footnotetext{
Corresponding author: Ivana Olivieri, IRCCS "Santa Maria Nascente" - Don Carlo Gnocchi ONLUS Foundation, Via Capecelatro 66, Milan, Italy. Tel.: +39 0240308173; E-mail: iolivieri@ dongnocchi.it
}

Pediatric rehabilitation therapists have always worked using a variety of off-the-shelf or specially designed objects and devices which have increasingly come to include computer solutions to train specific abilities. The appeal of easy-to-use, interactive videogame consoles based on detecting actual users' movements rather than pressing keys, has led a number of therapists to include them in their rehabilitation protocols. A proof of this evolution can be the recent introduction of the neologism Wiihabilitation, which derives from the use of Nintendo's Wii console (Nintendo, Kyoto, Japan) for rehabilitation activities [3]. Using commercially available consoles has many advantages (appeal, cost, and ease of use by therapists and patients), and although recent reviews indicate lack of large-scale studies and randomised controlled trials $[4,5]$, there is an increasing evidence of their positive effects $[6,7]$. 
However, these consoles are designed for the entertainment of average young people with normal abilities, and they have some limitations when used for rehabilitation purposes. First of all, they are usually not tailored to children with motor or cognitive impairments [8], as the videogames they run cannot be adapted to the specific needs and abilities of impaired children [8,9], and also because each console relies on its specific controllers (i.e. Nintendo WiiMote and Balance Board, Sony EyeToy, Microsoft Kinect). Moreover, as the goal of videogame players is to maximize the score, we speculate that impaired children may use compensatory movements and postures that could negatively affect the rehabilitation process. Thirdly, the measurements acquired by the sensing devices are sealed into the systems, and cannot be transferred and used elsewhere to assess rehabilitation progress.

Another approach has seen the development of more sophisticated Information and Communication Technologies (ICT) solutions specifically designed for rehabilitation $[8,10,11]$. These are often expensive and difficult to use, and their poor and not-so-challenging multimedia content makes them less appealing to children than traditional videogames.

Finally, although a number of architectures have recently been developed as commercial products/services (i.e. Neuro@home [12], VirtualRehab, etc.), or for research purposes (i.e. Rewire [13], Riablo [14] and Rehab@home [15], etc.), they do not seem to be particularly suitable for PR. Thus their routine use sometimes requires specific technical skills.

The term Web 2.0 refers to the current revolution in Internet technology. The contents of the original World Wide Web (Web 1.0) were defined a priori and presented to users in a fixed and unchangeable manner, whereas Web 2.0 is based on new interactive architectures that have opened the way to a number of interactive services, including the "social networks". Knight et al. [16] suggested using the name "Physical Therapy 2.0" to describe the innovative approach that combines traditional rehabilitation principles and the use of specifically designed ICT solutions. Hence, we propose the term "Pediatric Rehabilitation 2.0", where the main difference from the traditional approach is that computers play a key role in the rehabilitation process, thus extending their use beyond usual training software programs (i.e. cognitive training [17]) or the simple use of videogame consoles [6]. This may be accomplished by exploiting the interactivity and engagement of videogames as key factors to sustain a greater involvement of children, while increasing treatment efficacy.
Generally speaking, the computer-assisted approach is gaining widespread interest and popularity in the rehabilitation field due to the wide-spread diffusion of mobile and computer-based devices. However, since there is still a lack of strong evidence of its efficacy, due to the heterogeneity and small sample size of the available studies, it is important to address the issue appropriately, with the aim of bridging the gap between research efforts and clinical practice.

There is a real need to design solutions that can become a routine part of PR, while avoiding the pitfalls of misusing technology by becoming either "technologydriven" or "technology-reluctant". To cope with this challenge which requires the appropriate balance of new technologies and PR principles, we created a Computer-Assisted REhabilitation (CARE) Lab, a place where to study innovative approaches, we referred to as "Pediatric Rehabilitation 2.0". Thus, the aim of this paper is to describe the process that led us to design, build and start using this lab, and the keywords that guided us in this journey.

\section{Methodology and procedure}

In this section, the two main methodological issues related to the novel "Pediatric Rehabilitation 2.0" approach will be described as follows: 1) the theoretical framework and 2) the operative steps. These issues derived from our previous experience using an advanced ICT system (VRRS, from Khymeya, Padua, Italy) that, despite very interesting albeit preliminary results [10], revealed intrinsic limitations with respect to the broad spectrum of rehabilitation needs of children admitted to the Neuropsychiatric and Rehabilitation Unit. After a survey of the commercially available solutions, we realized there was a need to build a place specifically designed for a computer-assisted rehabilitation, by I) developing a suitable software architecture to support a variety of exercises, II) using various types of sensors and III) offering the possibility (in perspective) to provide multiple and differentiated feedback to the treated children.

\subsection{The theoretical framework}

Four basic principles (Ecological, Personalized, Interactive, Quantitative) guided the development of the CARE-Lab. The acronym EPIQ encapsulates these concepts. 
E - The potential of ecological approach in rehabilitation has been explored in previous studies [18-20], and stressed in a recent paper [21]. According to Classification of Functioning, Disability and Health (ICF), $\mathrm{PR}$ in addition to an improvement in in-lab motor or cognitive performances should also increase the children's personal, social and communicational autonomy, and their participation in their own environments [22]. Hence, beyond the enhancement of a single movement or a single cognitive function, PR should promote the transfer of the improvements achieved to other significant daily life activities. Consequently, rehabilitation interventions should foster engagement in functional tasks by taking into account the fundamental principle of the "ecological approach" which considers that individuals and their environment are not separate but mutually related [23].

$\mathbf{P}$ - It has long been known that the rehabilitation process not only needs to consider children's specific ability in relation to each individual clinical picture and their stage of development, but also their condition and attitude at the time of treatment. Therapists should tailor and modulate their intervention to enhance patients' motivation by keeping them in their "zone of proximal development" (i.e. "the distance between the actual developmental level as determined by independent problem solving and the level of potential development as determined through problem solving under adult guidance or in collaboration with more capable peers" [24]), and ultimately improve their learning pace [25].

I - One of the key factors for an effective rehabilitation in children is to attract and keep their attention throughout each session, particularly when the proposed exercises are long lasting or repetitive. In comparison with traditional rehabilitation, a computermediated approach using advanced ICT techniques such as Virtual Reality (VR) and sensors can offer a richer range of inputs related to body movements, gaze and voice and a broader range of visual, acoustic and haptic feedbacks, thus offering a higher interactivity level less dependent from child's abilities.

$\mathbf{Q}$ - Technology allows collecting quantitative information of a child's performance by measuring movements, posture, timing, and other data useful to monitor the improvements during the rehabilitation treatment. The quantitative monitoring during the rehabilitation sessions opens up new perspectives for clinicians and therapists who can obtain more detailed and continuous view of the rehabilitation process. Moreover, the information collected by instruments is almost op- erator independent and clinicians and therapists can focus on their patients and the interaction with them, thereby limiting the burden of measuring patients' performances.

\subsection{Operative steps: the VITAMIN platform and the CARE lab}

Based on the conceptual framework described above, the Computer-Assisted Rehabilitation (CARE) Lab has been recently developed and is operating at our institution. As previously mentioned, two steps were guiding our final goals: the need to develop a suitable software, given the lack of existing solutions capable of satisfying all of our requirements in terms of flexibility, openness, expandability and quantitative approach, and the need for physical place where the software platform can be operated according to EPIQ principles.

The first step was to design and develop a novel software architecture (see Fig. 1), we called VIrtual realiTy plAtform for Motor and cognItive rehabilitatioN (VITAMIN). The main features of this architecture had to match the following requirements to:

a) allow the collection of quantitative information concerning the activities performed by the patients;

b) support the concept of a personalized set of activities by decoupling the information needed to play the virtual game from the actual measured input, thus allowing a free and customizable combination of movements to be detected by one or more sensors;

c) accomplish a "videogame approach" in terms of graphic design (see Fig. 2), interaction sequences, music and sounds, using a videogame engine (Unreal Engine, Epic Games, Cary, NC, USA);

d) be designed for future extension to the home and ambulatory settings;

e) be controlled by a simple web interface that can be operated by a therapist using a tablet.

The second step was to design and build the CARE Lab as a tangible space. As shown in Figs 3 and 4 the lab, equipped with the hardware and specifically designed software to deliver motor and cognitive exercises in a virtual environment, is divided into three main rooms: a) a "hi-tech" room, b) a "low-tech" room, and c) a control room.

The "hi-tech" room is meant to provide the richest experiences for computer-assisted PR. It has been carefully designed around the child and therapist both vi- 


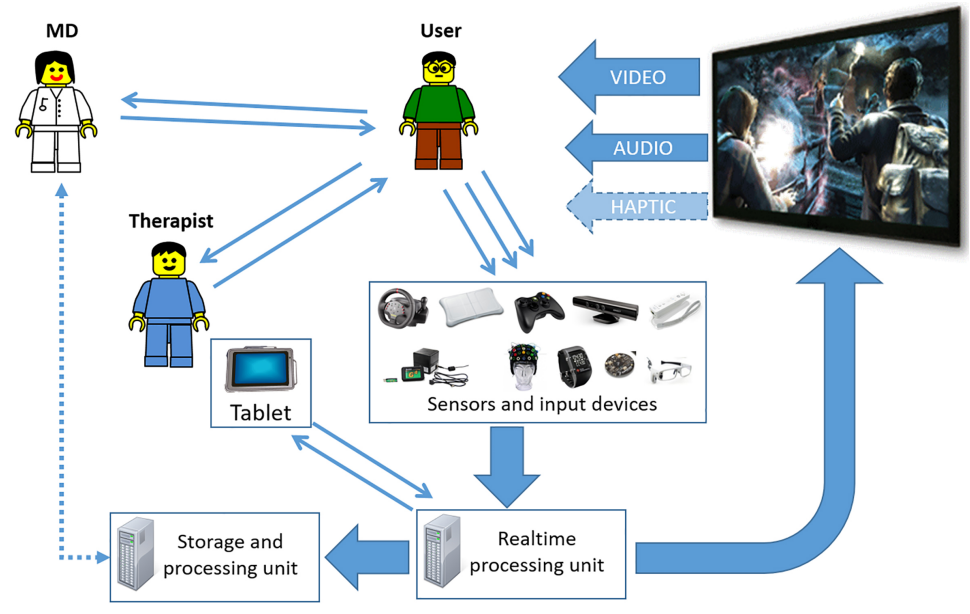

Fig. 1. An overview of VITAMIN software: the child's movements, acquired by a number of sensing devices (i.e. videogame handles, Kinect, more sophisticated devices, etc.) is translated into digital information. This information is then used for two purposes: it is processed within the game activity to calculate the audio/video (and haptic in the future) biofeedback to be presented to the user, while it is processed and stored for further off-line analysis. The therapist can adapt the activities in real-time through a tablet, while the clinician can supervise off-line the entire rehabilitation process by observing the data collected in the sessions.

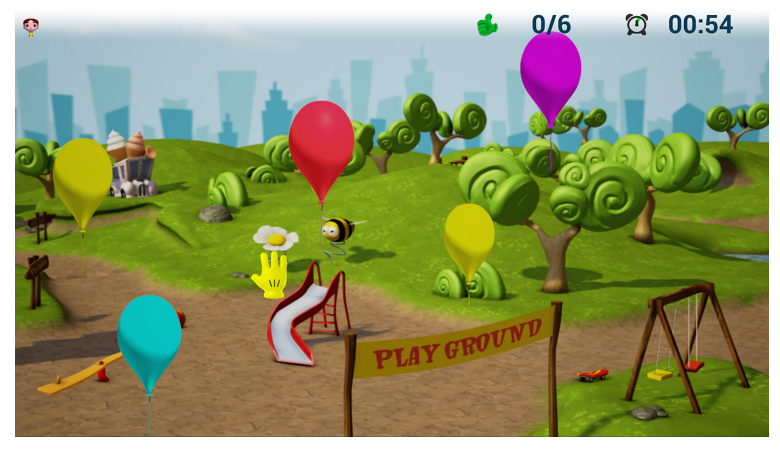

Fig. 2. An example of game activity: the "blow the balloons game".

sually and acoustically. There are five visually distinct areas: two side walls covered with colorful cartoonlike playground scenes; a frontal white screen that covers almost the entire field of view of the child looking forward; a rear wall in which a two-way mirror is hidden in the picture of an ice-cream van; the floor on which it is possible to project images and videos. The front screen projector has high-resolution (full HD) and lumen capabilities in order to ensure sharp and bright images. The room has been acoustically equipped in order to minimize reverberations: acoustic panels are hidden behind the wall coverings and the screen. High-fidelity sound is ensured by a hi-end amplifier (A-3030, Yamaha, Hamamatsu, Japan) and a Dolby ${ }^{\circledR} 7.1$ system consisting of seven loudspeakers (LCR950, MK Sound, Karlslunde, Denmark) and a subwoofer (V8, MK Sound). It is also possible to adjust the lighting (either in terms of intensity and color) of the room in order to help the children concentrate on the proposed activity. The overall visual and acoustic design ensures that they can immerse themselves in the exercise while clearly hearing the therapist. Side panels are also designed to hide the electronic systems and their cabling, thus reinforcing the feeling of being in a playroom rather than in a cold laboratory.

The "low-tech" room has been designed to test the suitability of appropriate selection of devices and technologies used in the hi-tech room before they can be transferred to children's homes to provide continuity of care.

The "control room" houses the core multimedia and computing capabilities of the CARE Lab. It has been built with two goals: clinicians, therapists, students and possibly caregivers can observe the session unobtrusively, and engineers and scientists can develop and analyze their technological solutions. The two-way mirrors located between the control room and the hi- and low-tech rooms make it possible to observe the session from outside. In addition, loudspeakers relay the sounds picked up by special microphones. This audio/video information can also be recorded for subsequent clinical and/or educational purposes after having obtained the necessary authorization by caregivers.

We are also considering some improvements of VITAMIN and CARE Lab as described below:

\subsubsection{Increasing the number and kind of games}

VITAMIN has the capacity to easily create increasingly difficult tasks. There is still the need to continue 


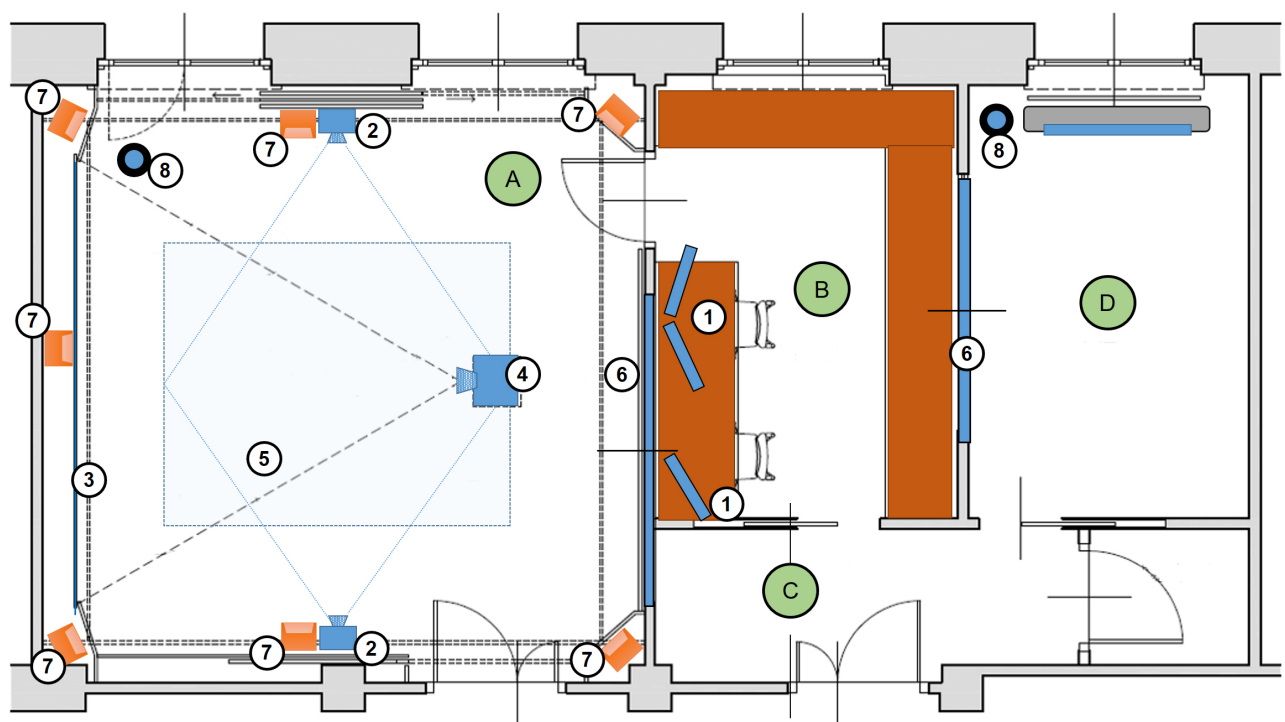

Fig. 3. CARE Lab layout: (A) the hi-tech room equipped with advanced multimedia and multimodal solutions; (B) the control room; (C) the observation room from which it is possible to see the activities being carried out through a two-way mirror; (D) the low-tech room equipped with the low-cost systems and devices needed to pre-evaluate home-based rehabilitation. CARE Lab. equipment: (1) main computer; (2) ceiling projectors for floor images; (3) frontal screen; (4) main ceiling projector; (5) floor projection area; (6) two-way mirrors; (7) 7.1 Dolby ${ }^{\circledR}$ HiFi Surround loudspeakers; (8) session-monitoring cameras.
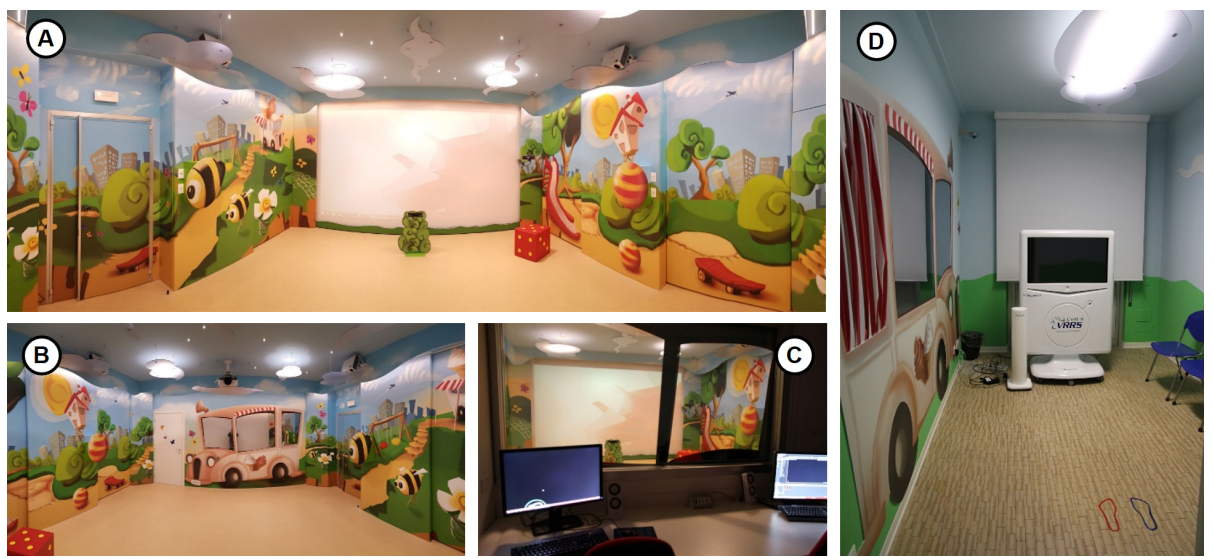

Fig. 4. Views of the CARE Lab: (a) the hi-tech room with frontal screen and side panels; (b) the back of the hi-tech room with the ice-cream van hiding the two-way mirror; (c) the hi-tech room seen from the control room; (d) the low-tech room.

to develop or integrate new engaging activities as suggested by recent papers $[4,26]$. Providing new stimuli during the rehabilitation process helps to sustain the motivation.

\subsubsection{Integrating other software solutions}

We developed VITAMIN in an attempt to overcome the main limitations of the videogame consoles used in rehabilitation, and we would like to extend its functionalities not only by developing new software of our own, but also by integrating other interesting and us- able solutions (i.e. Ripple, an open source software developed by Microsoft professionals - see Fig. 5c and d).

\subsubsection{Extending the lab's sensing capabilities}

It is essential to acquire other measurements to complement those sensed by Kinect, such as fine finger movements, pronation-supination, eye movements, interactions in balancing, touching/grasping/carrying objects, etc. Some examples are the Leap Motion controller (Leap Motion, San Francisco, USA) for finger 

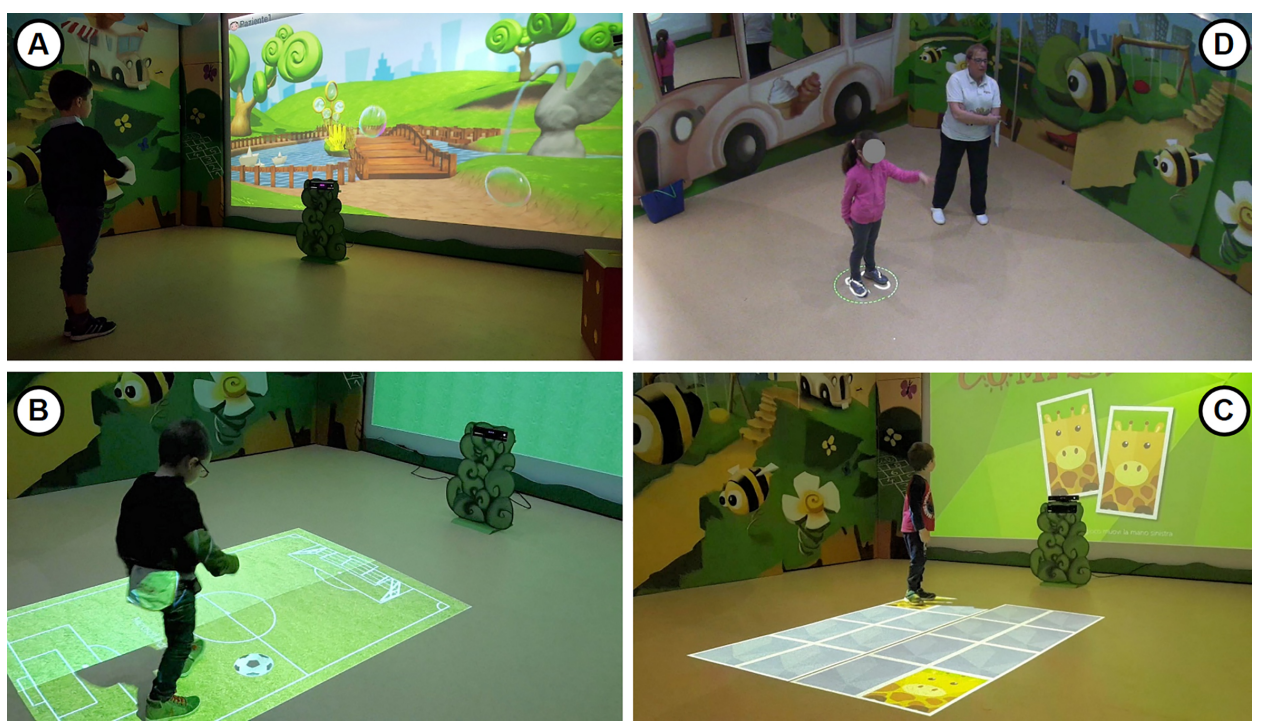

Fig. 5. The hi-tech room in use: (a) a game being played using VITAMIN software, with the images projected onto the frontal screen; (b) a typical rehabilitation session; (c, d) other exercises performed using images projected onto the floor.

tracking, or small wearable inertial devices for the detailed detection of the hand position. Another possibility would be to instrument the floor to detect and measure pressure areas, which could be combined with current kinematic measurements to enrich the assessment of a child's full body motion (for example when walking). Eye-tracking devices such as Tobii EyeEX (Tobii AB, Danderyd, Sweden) will be evaluated, especially in the perspective of administering exercises in the cognitive rehabilitation area. Finally, we will consider small wireless solutions to acquire physiological signals (EEG, EMG, Electrocardiography, Galvanic Skin Response, etc.).

\subsubsection{Extending the actuators and biofeedback}

We are currently using video feedback through the front main screen and to a certain extent some of the audio capabilities of the audio-video system. However, children who may benefit from a computer-assisted rehabilitation approach may also have visual, acoustic or sensorimotor limitations that could hamper the treatment's efficacy. For this reason, we will be studying some possible solutions for appropriate feedbacks using suitable haptic (force and tactile) and directional audio feedbacks. We will also consider the possible involvement of the assistive technologies in the rehabilitation process carried out in the CARE Lab in all cases when children need to complement their abilities with suitable orthoses, prostheses or other instruments.

\subsection{An example of upper limb rehabilitation session in the CARE Lab}

For a typical session involving upper limb rehabilitation activities, the therapist brings the child into the room and uses a tablet to set up the rehabilitation game and parameters such as the limb to be used, virtual targets' speed, the length of the game, etc. (see Fig. 5a and $b$ ). The therapist then starts the calibration procedure by asking the child to move his/her arm making the biggest circles he/she can draw in the air, moving the arm on the frontal plane, while standing in a fixed position on the floor. This procedure is essential in order to ensure that the gaming experience is tailored to the child's abilities by identifying the actual selected limb's working volume for that session. The calibration procedure can be repeated during the session if necessary, and can be performed also selecting the healthy limb for further intra-subject analysis.

As the stimuli are automatically projected on the screen or floor and transmitted through the loudspeakers by the software running on the workstations, the therapist can concentrate on the child's movements without the burden of recording times or geometric information, all of which are seamlessly captured by sensing devices. At the end of each game and session, some results (i.e. overall scores, the number of targets hit, the number of errors, etc.) are reported to the therapist on the tablet which can also be used to record other notes. 


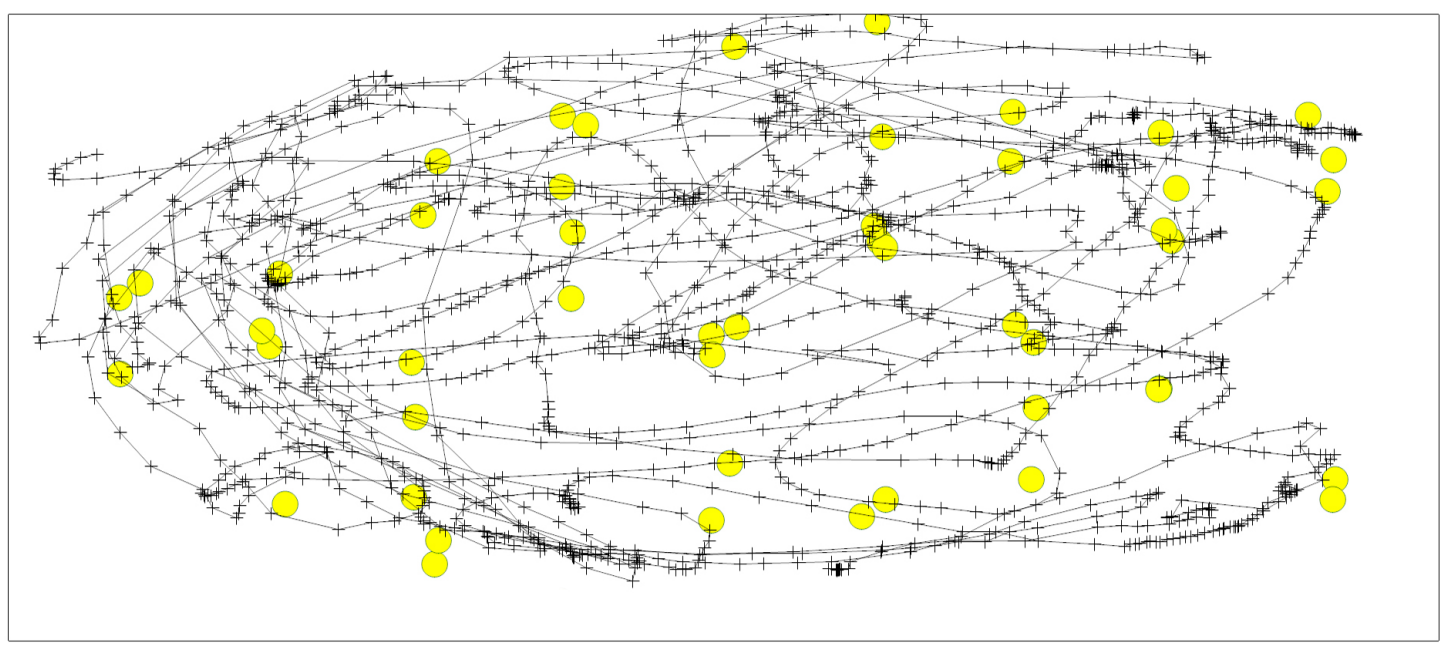

Fig. 6. An example of hand's trajectory during an exercise; the yellow circles indicate where the virtual hand (moved by the child) hit the virtual bubbles.

The quantitative information acquired (i.e. limb movements see Fig. 6, etc.) is made available on workstations for post-session processing in order to evaluate trends in the child's rehabilitation process more precisely, by extracting some parameters such as trajectory length, number of hits and their distribution on the screen, etc. In addition, we are also studying a number of more sophisticated indexes derived from gait and other analyses, and the graphical representation of data that might be useful for an overall assessment of the quality of movement. This is absolutely necessary as it helps clinicians and therapists to personalize their future interventions and make their interventions more effective. It is to be noted that this still requires good multidisciplinary research, as it is not easy to merge the typically qualitative considerations expressed by therapists, clinicians and caregivers together with this quantitative information into the same framework.

The pre and post-rehabilitation assessments consist of the functional evaluation of paretic upper limb using the "Melbourne Assessment of Unilateral Upper Limb Function Scale" (MA2), passive and active shoulder and elbow range of motion (ROM) and of a kinematic (optoelectronic) and EMG analysis. The ABILHANDKids questionnaire is administered to parents in order to measure their children's ability to manage daily activities that require the use of the upper limbs.

\section{Results}

A pilot study on subjects aged 7-14 years with congenital hemiparesis is currently ongoing. The rehabil- itation protocol consists of ten weekly sessions, with rehabilitation games specifically designed and developed to improve frontal and lateral reaching with the paretic upper limb and other exercises for pronationsupination and tracking movements.

Figure 6 shows an example plot of the trajectory of a child's involved hand during a simple exercise of moving a virtual hand with the aim of bursting all the virtual bubbles that appear on the screen.

Preliminary results regarding a small group of six subjects show: an overall increase in MA2 scores, especially the median improvement in "movement range" (from $61.1 \%$ to $81.1 \%$ ), "dexterity" (from $52.6 \%$ to $65.8 \%$ ), "fluency" (from $52.4 \%$ to $62 \%$ ), and "precision" (from 92\% to 96\%); an increase in passive and active ROM, with mean passive and active shoulder flexion respectively improving from $151.2^{\circ}$ to $161.7^{\circ}$ and from $144.2^{\circ}$ to $152.3^{\circ}$, mean passive and active shoulder extension respectively improving from $36.7^{\circ}$ to $42.5^{\circ}$ and from $29.2^{\circ}$ to $33.7^{\circ}$; and an overall increase in upper limb motor performance as revealed by the ABILHAND-Kids questionnaire. Furthermore the parents of four of the children reported improvements in "Zipping up a jacket", "Putting on a backpack", and "Washing the upper body".

Although these are only preliminary results from a very small sample of children, they are encouraging at both functional and assessment levels, and also in terms of the activities of daily life (ADL). They are even more interesting bearing in mind that they were obtained after a small number of weekly sessions carried out over a limited period of time (2-3 months). 


\section{Discussion and conclusion}

We are in the middle of a scientific, clinical and social transformation driven by the pervasive spread of ICT devices and systems (smartphones, tablets, wearables, etc.), the effects of which will also profoundly influence the entire approach to rehabilitation. These solutions may not only affect traditional rehabilitation techniques, but could also lead to a new and intrinsically quantitative PR paradigm based on increased inner motivation and patient engagement.

Pediatric Rehabilitation 2.0 is thus characterized, in our opinion, by its transition from the usual three-step approach (pre-intervention assessments, rehabilitation, and post-intervention assessments) to a more continuous one in which the data collected during the rehabilitation process can be used continuously to assess and adapt the process itself.

This paper briefly describes some important theoretical aspects and the experience so far gained in the field, which we have summarized in the acronym EPIQ (Ecological, Personalized, Interactive, and Quantitative). Thanks to this experience which has highlighted both the complexity and the potentialities of this new approach, we are conscious of being at the beginning of a new journey that will require a number of open questions to be addressed and, possibly, answered.

1. For a thorough evaluation of the computer-assisted approach's efficacy it is necessary to clarify how much of the rehabilitation work carried out in a virtual or artificial environment can support the real transfer of newly learned skills to ADL. Moreover, it should be carefully assessed for how long, whether and to what extent the "smartification" of objects (i.e. transforming usual objects into connected and interactive ones) and/or the surrounding environment can contribute to facilitate such a transfer.

2. The growing shift to home- and ambulatorybased rehabilitation will likely lead to higher intensity and reasonably greater efficacy, in addition to empowering caregivers, families and teachers in the rehabilitation process. Additionally, it can lay the foundation for the creation of communities that can further help to support everyday family life. However, some issues need to be considered:

a. extending the rehabilitation process to the home setting requires appropriate technical solutions that take into account the distance, and the physical absence of the therapist, while assuring a continuous overview of the process by therapists and clinicians.

b. activities need to be challenging in order to keep the children in the zone of proximal development during most of the process which may require software capable of incorporating some kind of auto-tuning on the basis of a child's performance.

c. data collected must be used respecting the patients' privacy in addition to observing laws governing data protection. This is because the growing use of distributed and cloud-based repositories and services is not only a great opportunity for home-based rehabilitation, but also a potential threat that has to be considered.

3. It is also essential to ensure that the processing of the acquired information provides compact indices that support healthcare professionals and caregivers in doing their jobs. The potentially enormous amounts of data acquired during a single exercise can become useful only if it is appropriately processed before being (numerically/graphically/acoustically) reported in realtime to the therapists and patients, and off-line to therapists and clinicians. Furthermore, there will be a need to adequately study the relationship between this amount of acquired data and the usual clinical scales, which instead are usually evaluated only a few times (two/three in the vast majority of cases).

4. In order to take full advantage of this novel approach, it is mandatory to create multidisciplinary teams suitable to work together to study appropriate procedures and new services. Also, it is necessary to ensure appropriate training for clinicians, therapists and caregivers.

Like the recent literature [4], these considerations underline the increasing need for further and larger studies of the ways in which the increasingly pervasive presence of technology is likely to reshape PR in the near future.

\section{Acknowledgments}

The authors would like to thank the Elena Pajan Parola Foundation and the Associazione Zorzi per le Neuroscienze for financially supporting the development of VITAMIN and the CARE lab. Our particular thanks also go to Paolo Lazza, the architect who 
helped to translate ideas into tangible spaces; Massimo Comuzio, Diego Cortassa and Maurizio Casini for the software development; and the members of Studio Bozzetto \& Co for their work on the design of the graphic elements used in VITAMIN. We would like also to thank Dr. Roberto Spreafico for his precious scientific mentoring role.

\section{Conflict of interest}

The authors have no conflict of interest to report.

\section{References}

[1] Assembly UG, Convention on the Rights of Persons with Disabilities: resolution/adopted by the General Assembly. 2007.

[2] Bamm EL and Rosenbaum P. Family-centered theory: origins, development, barriers, and supports to implementation in rehabilitation medicine. Arch Phys Med Rehabil, 2008; 89(8): p. $1618-24$

[3] Anderson F, Annett M and Bischof WF. Lean on Wii: physical rehabilitation with virtual reality Wii peripherals. Stud Health Technol Inform, 2010; 154: p. 229-34.

[4] Ravi DK, Kumar N and Singhi P. Effectiveness of virtual reality rehabilitation for children and adolescents with cerebral palsy: an updated evidence-based systematic review. Physiotherapy, 2017.

[5] Taylor MJ et al., Activity-promoting gaming systems in exercise and rehabilitation. J Rehabil Res Dev, 2011; 48(10): p. 1171-86.

[6] Parry I et al., A Pilot Prospective Randomized Control Trial Comparing Exercises Using Videogame Therapy to Standard Physical Therapy: 6 Months Follow-Up. J Burn Care Res, 2015; 36(5): p. 534-44.

[7] Andrysek J et al., Preliminary evaluation of a commercially available videogame system as an adjunct therapeutic intervention for improving balance among children and adoles cents with lower limb amputations. Arch Phys Med Rehabil, 2012; 93(2): p. 358-66.

[8] Green D and Wilson PH. Use of virtual reality in rehabilitation of movement in children with hemiplegia - a multiple case study evaluation. Disabil Rehabil, 2012; 34(7): p. 593604.

[9] Rowland JL, et al., Perspectives on Active Video Gaming as a New Frontier in Accessible Physical Activity for Youth With Physical Disabilities. Physical Therapy, 2016; 96(4): p. 521532.
[10] Olivieri I et al., Rehabilitation of children with hemiparesis: a pilot study on the use of virtual reality. Biomed Res Int, 2013 2013: p. 695935.

[11] Brutsch K et al., Virtual reality for enhancement of robotassisted gait training in children with central gait disorders. J Rehabil Med, 2011; 43(6): p. 493-9.

[12] Gagliardo P, et al., Neuro@home: A software platform of clinically designed videogames specifically designed for the motor rehabilitation of stroke patients. Brain Injury, 2014; 28(5-6): p. 827-827.

[13] Mainetti, R., et al., Duckneglect: video-games based neglect rehabilitation. Technol Health Care, 2013; 21(2): p. 97-111.

[14] Leardini A, et al., Validation of the angular measurements of a new inertial-measurement-unit based rehabilitation system: comparison with state-of-the-art gait analysis. J Neuroeng Rehabil, 2014; 11: p. 136.

[15] Faria C, Silva J and Campilho A. Rehab@home: a tool for home-based motor function rehabilitation. Disabil Rehabil Assist Technol, 2015; 10(1): p. 67-74.

[16] Knight E et al., Physical therapy 20: leveraging social media to engage patients in rehabilitation and health promotion. Phys Ther, 2015; 95(3): p. 389-96.

[17] Karch, D et al., The efficacy of cognitive training programs in children and adolescents: a meta-analysis. Dtsch Arztebl Int, 2013; 110(39): p. 643-52.

[18] Ahl, L.E., et al., Functional therapy for children with cerebral palsy: an ecological approach. Dev Med Child Neurol, 2005 47(9): p. 613-9.

[19] Gibson BE et al., Revisiting therapy assumptions in children's rehabilitation: clinical and research implications. Disabil Rehabil, 2009; 31(17): p. 1446-53.

[20] Ketelaar $\mathrm{M}$ et al., Effects of a functional therapy program on motor abilities of children with cerebral palsy. Phys Ther, 2001; 81(9): p. 1534-45.

[21] Vaz DV et al., Towards an ecologically grounded functional practice in rehabilitation. Hum Mov Sci, 2017; 52: p. 117-132.

[22] Organisation WH, International Classification of Functioning, Disability and Health - Children and Youth version (ICF-CY), WH. Organisation, Editor. 2007.

[23] Gibson, J.J., The ecological approach to visual perception. 1979; Boston: Houghton Mifflin.

[24] Vygotsky, L.S., Interaction between learning and development, in Mind in society: The development of higher psychological processes, VJ.-S.M. Cole, S. Scribner and E. Souberman Editor. 1978, Harvard University Press: Cambridge, MA. p. 79-91.

[25] Lyons BG, Defining a child's zone of proximal development: evaluation process for treatment planning. Am J Occup Ther, 1984; 38(7): p. 446-51.

[26] Weiss PL, Tirosh E and Fehlings D. Role of virtual reality for cerebral palsy management. J Child Neurol, 2014; 29(8): p. 1119-24. 